\title{
Tracheostomy in Postoperative Pediatric Cardiac Surgical Patients-The Earlier, the Better
}

\author{
Chalattil Bipin ${ }^{1}$ Manoj K. Sahu ${ }^{1}$ Sarvesh P. Singh ${ }^{1}$ Velayoudam Devagourou ${ }^{1}$ \\ Palleti Rajashekar ${ }^{1}$ Milind P. Hote ${ }^{1}$ Sachin Talwar ${ }^{1}$ Shiv K. Choudhary ${ }^{1}$ \\ ${ }^{1}$ Intensive Care for Cardio Thoracic Vascular Surgery, Cardio Neuro \\ Address for correspondence Manoj Sahu, MD, Intensive Care \\ Center, All India Institute of Medical Sciences, New Delhi, India \\ for CTVS, CNC, All India Institute of Medical Sciences, New Delhi \\ 110029, India (e-mail: drmanojsahu@gmail.com).
}

J Card Crit Care 2021;5:114-123.

\section{Abstract \\ Keywords \\ - pediatric cardiac surgery \\ - intensive care \\ - tracheostomy \\ - prolonged mechanical ventilation}

Objectives This study was aimed to assess the benefits of early tracheostomy (ET) compared with late tracheostomy (LT) on postoperative outcomes in pediatric cardiac surgical patients.

Design Present one is a prospective, observational study.

Setting The study was conducted at a cardiac surgical intensive care unit (ICU) of a tertiary care hospital.

Participants All pediatric patients below 10 years of age, who underwent tracheostomy after cardiac surgery from January2019 to december2019, were subdivided into two groups according to the timing of tracheostomy: "early" if done before 7 days or "late" if done after 7 days postcardiac surgery.

Interventions ET versus LT was measured in the study.

Results Out of all 1,084 pediatric patients who underwent cardiac surgery over the study period, 41 (3.7\%) received tracheostomy. Sixteen (39\%) patients underwent ET and 25 (61\%) underwent LT. ET had advantages by having reduced risk associations with the following variables: preoperative hospital stay $(p=0.0016)$, sepsis $(p=0.03)$, high risk surgery $(p=0.04)$, postoperative sepsis $(p=0.001)$, C-reactive protein $(p=0.04)$, ventilator-associated pneumonia (VAP; $p=0.006)$, antibiotic escalation $(p=0.006)$, and antifungal therapy $(p=0.01)$ requirement. Furthermore, ET was associated with lesser duration of mechanical ventilation ( $p=0.0027$ ), length of ICU stay (LOICUS; $p=0.01$ ), length of hospital stay (LOHS; $p=0.001)$, lesser days of feed interruption $(p=0.0017)$, and tracheostomy tube change $(p=0.02)$. ET group of children, who had higher total ventilation-free days $(p=0.02)$, were decannulated earlier $(p=0.03)$ and discharged earlier $(p=0.0089)$.

Conclusion ET had significant benefits in reduction of postoperative morbidities with overall shorter mechanical ventilation, LOICUS, and LOHS, better nutrition supplementation, lesser infection, etc. These benefits may promote faster patient convalescence and rehabilitation with reduced hospital costs. published online March 8, 2021
DOI https://doi.org/

10.1055/s-0041-1723749

ISSN 2457-0206. (c) 2021. Official Publication of The Simulation Society (TSS), accredited by International Society of Cardiovascular Ultrasound (ISCU).

This is an open access article published by Thieme under the terms of the Creative Commons Attribution-NonDerivative-NonCommercial-License, permitting copying and reproduction so long as the original work is given appropriate credit. Contents may not be used for commercial purposes, or adapted, remixed, transformed or built upon. (https://creativecommons.org/licenses/by-nc-nd/4.0/) Thieme Medical and Scientific Publishers Pvt. Ltd., A-12, 2nd Floor, Sector 2, Noida-201301 UP, India 


\section{Introduction}

Last two decades has seen tremendous growth and progress in cardiac surgery in infants and children because of advancements in perfusion, postoperative care, newer anesthetics, and refinements in surgical techniques, so as the increased number of complex congenital cardiac repairs. Many of such children require prolonged mechanical ventilation (MV) and its associated morbidities, as well as mortality postoperatively. For those who require prolonged MV, endotracheal tube is frequently replaced by a tracheostomy tube for smooth liberation from MV and to improve patient comfort and survival. Tracheostomy is seen to be commonly performed in children undergoing high-risk complex surgical procedures; postsurgery cardiac complications, such as severe ventricular dysfunction, low cardiac output, residual lesions, and sepsis; and those with syndromic association, airway issues, etc. ${ }^{1}$

The various advantages of tracheostomy over endotracheal intubation include greater patient comfort with secure airway that facilitates pulmonary toileting, rapid discontinuation of sedatives which allows these children to remain awake, communicative, oral feeding, and hemodynamic stability with easier and earlier weaning from MV and fewer ventilator-associated events. However, complications related to tracheostomy, for example, bleeding, hypoxia, esophageal injury, tracheal stenosis, tracheal granulomas, and death, are still seen in few.

About $10 \%$ of patients who required MV for longer than 10 days are tracheostomized; however, there is significant variability among institutional protocols. Numerous studies undertaken in general intensive care units (ICUs) have shown that early tracheostomy (ET) can reduce patient length of ICU stay (LOICUS), length of hospital stay (LOHS), duration of MV, and sedatives use which have large cost implications. ${ }^{2-4}$ There is no consensus till yet regarding optimal timing to perform tracheostomy, thus there is no clear definition of ET. American 2001 consensus document advocates the use of ET in patients who require prolonged MV but makes no recommendations regarding timing of the procedure. ${ }^{5}$ The Indian Society of Critical Care Medicine (ISCCM) expert panel practice recommendations has defined ET as up to and including 7 days of endotracheal intubation and MV. ${ }^{6}$

Increase in number of complex pediatric cardiac surgical repairs in last two decades has changed the practice of ICU management for faster patient convalescence, rapid turnover and better survival till hospital discharge. ${ }^{1}$ So tracheostomy as an ICU procedure comes in handy, supporting the tiny hearts recover after major surgical insults in a time bound fashion, further acute complications and subsequent hospital readmissions are also reduced. ${ }^{7}$ Contrary to the positive arguments favoring tracheostomy in cardiac surgical ICU (CSICU), performing ET postoperatively has been flawed with apprehensions. ${ }^{8,9}$ Very few studies are available on the benefits of ET compared with LT in pediatric cardiac surgical patients. We thus studied the advantages of ET in such subset of patient's postcardiac surgery in a tertiary care institute.

\section{Methods}

We conducted a prospective study on all pediatric cardiac surgical patients of $\leq 10$ years of age who underwent tracheostomy postoperatively from January 2019 to December 2019 in the CSICU of a tertiary care institute. This study was approved by the Institute Ethics Committee.

Informed consent was taken from parents of the participants. Those who were tracheostomized before surgery, as well as those who did not wish to participate, were excluded from the study.

The decision to perform tracheostomy was made by the intensivist and cardiac surgeon together based on clinical criteria including inability to wean off MV, neurological injury, sepsis, airway abnormality, residual cardiac lesions, etc.

The timing of tracheostomy was based on the hemodynamic stability, the anticipated course in the ICU included morbidities and was done after reviewing various clinical and laboratory parameters.

All the tracheostomies in these children were performed surgically at bedside in the CSICU. Enteral feeding was interrupted for 6 hours for the procedure. Choice of cuffed or uncuffed tube was based on the age of the patient. Antibiotics were continued as per the unit protocol based on individual patient profile. Posttracheostomy weaning of MV was continued in a stepwise manner. Enteral nutrition was continued through Ryles tube till child started taking orally. Patients were discharged from ICU to ward after a mandatory period of 48 hours of unsupported ventilation, minimal secretions, and adequate gas exchange. We preferably tried to decannulate our patients in the ICU before discharge.

Data of all our study participants including pre-, intra-, and postcardiac surgery, peritracheostomy, and outcome variables were collected. Time to tracheostomy was defined as "early" if performed before 7 days or "late," if done after 7 days postcardiac surgery. Patients were classified into two groups ET and LT. Both groups were compared with respect to patient demographics, surgical characteristics, outcomes, and postoperative complications.

\section{Definitions}

1. Underweight, wasting, and stunting are defined according to the National Centre for Health Statistics (NCHS) standards classification. ${ }^{10}$

2. High-risk cardiac surgery is defined according to risk adjustment for congenital heart surgery (RACHS) 1 surgical complexity score. (Score>2)

3. Acute kidney injury (AKI) is defined according to kidney disease improving global outcomes (KDIGO) criteria. ${ }^{11}$

4. Central nervous system (CNS) event: any change in the Glasgow coma score or any postoperative neurologic disorder (visual, cognitive, motor, or speech) documented and/or neurologic disability severely affecting day-to-day functioning which can be attributed to a CNS pathology or biochemical changes.

5. Sepsis is defined as per the sepsis-3 definition. ${ }^{12}$ 
6. Hospital-acquired infections (HAI) including catheter-associated urinary tract infection (CAUTI), catheter-related blood stream infection (CRABSI), ventilator-associated pneumonia (VAP), and surgical site infection (SSI) were defined according to the Centers for Disease Control and Prevention (CDC) definitions. ${ }^{13}$

\section{Statistical Analysis}

Statistical analysis was conducted with the use of SPSS version 20 (StataCorp, 4905, Lake way Drive, College Station, Texas, United States). Demographic and clinical variables are described as mean \pm SD for normally distributed continuous data, median (interquartile range) for skewed data and frequencies (\%) for categorical variables. Variables were compared using nonparametric Wilcoxon's rank-sum test for the continuous data and Fisher's exact test for categorical variables. A $p$-value of $<0.05$ was considered statistically significant.

\section{Results}

During the 1-year study period, 1,084 index pediatric cardiac operations were performed. Tracheostomy was performed in 41 (3.7\%) of these patients.

Details regarding comprehensive cardiac diagnoses, surgical procedures, RACHS1 score, etc in the study population are elaborated in - Table 1. Patient demography, anthropometric measurements, pre- and intrtaoperative variables are compared in - Table 2 .

The mean age of the 41 tracheostomized children was $3 \pm 4.1$ years (median, 1 year). Thirty (73\%) of these children were male and 11 (27\%) were female. Five children were syndromic, two had trisomy 21 , one child had thrombocytopenia absent radius (TAR) syndrome, one had Goldenhar's syndrome; and one with vertebral defect anal atresia cardiac defect tracheoesophageal fistula renal abnormality limb abnormality (VACTERL) association. Preoperative variables,

Table 1 Pediatric cardiac surgery patients who required tracheostomy postoperatively

\begin{tabular}{|c|c|c|c|c|c|}
\hline SI. no. & Diagnosis & Cardiac surgery & No of patients (\%) & RACHS 1 score & No of ET patients (\%) \\
\hline 1 & TOF & Total correction & $9(23.2)$ & 2 & $6(66.6)$ \\
\hline 2 & TOF pulmonary atresia & Conduit repair & $1(2.4)$ & 2 & $1(100)$ \\
\hline 3 & d-TGA & $\begin{array}{l}\text { Arterial switch } \\
\text { operation }\end{array}$ & $10(24)$ & 4 & $2(20)$ \\
\hline 4 & VSD & VSD closure & $3(7.2)$ & 2 & $3(100)$ \\
\hline 5 & SC TAPVC & TAPVC repair & $2(4.8)$ & 2 & $1(50)$ \\
\hline 6 & IC TAPVC & TAPVC repair & $2(4.8)$ & 4 & $0(0)$ \\
\hline 7 & AVSD & AVSD repair & $1(2.4)$ & 3 & $0(0)$ \\
\hline 8 & AVSD PAH & AVSD repair & $4(9.6)$ & 4 & $0(0)$ \\
\hline 9 & Partial AVSD PDA severe PAH & $\begin{array}{l}\text { PA band atrial } \\
\text { septectomy PDA } \\
\text { ligation }\end{array}$ & $1(2.4)$ & 3 & $0(0)$ \\
\hline 10 & Ebstein's anomaly & Cone repair & $1(2.4)$ & 5 & $0(0)$ \\
\hline 11 & Pulmonary atresia IVS & $\begin{array}{l}\text { Pulmonary valvot- } \\
\text { omy RVOT patch }\end{array}$ & $1(2.4)$ & 2 & $0(0)$ \\
\hline 12 & $\begin{array}{l}\text { Type- } 3 \text { patent truncus } \\
\text { arteriosus }\end{array}$ & $\begin{array}{l}\text { Truncus arteriosus } \\
\text { repair }\end{array}$ & $1(2.4)$ & 5 & $0(0)$ \\
\hline 13 & AP window & AP window repair & $1(2.4)$ & 2 & $1(100)$ \\
\hline 14 & IPAH severe PAH & Pott's shunt & $1(2.4)$ & 3 & $1(100)$ \\
\hline 15 & $\begin{array}{l}\text { TOF Pulmonary atresia hypo- } \\
\text { plastic main and left PA }\end{array}$ & $\begin{array}{l}\text { RMBTS followed by } \\
\text { BD Glenn }\end{array}$ & $1(2.4)$ & 3 & $1(100)$ \\
\hline 16 & VSD mesocardia small RV & BD Glenn & $1(2.4)$ & 2 & $0(0)$ \\
\hline \multirow[t]{2}{*}{12} & $\begin{array}{l}\text { d-TGA large inlet VSD pulmo- } \\
\text { nary atresia aortopulmonary } \\
\text { collaterals post-RMBTS/BD } \\
\text { Glenn/azygous vein ligation } \\
\text { post coil }\end{array}$ & Completion Fontan & $1(2.4)$ & 3 & $0(0)$ \\
\hline & Total & & $41(100)$ & & $16(39)$ \\
\hline
\end{tabular}

Abbreviations: AP, aortopulmonary; AVSD, atrioventricular septal defect; BD, bidirectional; ET, early tracheostomy; IC, infracardiac; IPAH, idiopathic pulmonary artery hypertension; IVS, intact ventricular septum; PA, pulmonary artery; PAH, pulmonary artery hypertension; PDA, patent ductus arteriosus; RACHS, risk adjustment for congenital heart surgery; RMBTS, right modified Blalock-Taussig shunt; RV, right ventricle; RVOT, right ventricular outflow tract; SC, supracardiac; TAPVC, total anomalous pulmonary venous connection; TGA, transposition of great arteries; TOF, tetralogy of Fallot; VSD, ventricular septal defect. 
Table 2 Comparison between the demography preoperative nutrition diagnosis preoperative and intraoperative characteristics between early tracheostomy and late tracheostomy

\begin{tabular}{|c|c|c|c|c|}
\hline SI. no. & $\begin{array}{l}\text { Clinical variables } \\
n(\%)\end{array}$ & $\begin{array}{l}\text { Early tracheostomy } \\
(n=16)\end{array}$ & $\begin{array}{l}\text { Late tracheostomy } \\
(n=25)\end{array}$ & $p$-Value \\
\hline 1 & Age in years (mean $\pm S D$ ) & $3.19 \pm 3.84$ & $2.89 \pm 4.28$ & 0.23 \\
\hline \multirow[t]{4}{*}{2} & Age categories (y) & & & \multirow[t]{4}{*}{0.19} \\
\hline & $\leq 1$ & $5(31)$ & $14(56)$ & \\
\hline & $>1-5$ & $8(50.3)$ & $6(24)$ & \\
\hline & $>5-10$ & $3(18.7)$ & $5(20)$ & \\
\hline 4 & Male & $10(62.5)$ & $20(80)$ & 0.19 \\
\hline 5 & Weight in kg (mean \pm SD) & $9.44 \pm 6.13$ & $9.02 \pm 7.87$ & 0.47 \\
\hline 6 & Underweight & $12(75)$ & $18(72)$ & 0.67 \\
\hline 7 & Height in cm (mean \pm SD) & $83.37 \pm 23.74$ & $80.12 \pm 30.76$ & 0.39 \\
\hline 8 & Stunting & $9(56.1)$ & $6(24)$ & 0.06 \\
\hline 9 & Wasting & $11(69)$ & $15(60)$ & 0.41 \\
\hline 10 & Pre-op hospitalization in days (mean \pm SD) & $2.18 \pm 2.68$ & $11.44 \pm 13.27$ & 0.001 \\
\hline 11 & Chromosomal anomaly & $2(12.6)$ & $3(12)$ & 0.65 \\
\hline 12 & Neuromuscular disorder & $0(0)$ & $1(4)$ & 0.61 \\
\hline 13 & Chronic lung disease & $0(0)$ & $1(4)$ & 0.61 \\
\hline 14 & Pre-op mechanical ventilation (MV) & $1(6.3)$ & $4(16)$ & 0.34 \\
\hline 15 & Previous surgery & $3(18.7)$ & $6(24)$ & 0.50 \\
\hline 16 & Pre-op sepsis & $1(6.3)$ & $9(36)$ & 0.03 \\
\hline 17 & Cyanotic & $13(81.2)$ & $20(80)$ & 0.62 \\
\hline 18 & Increased pulmonary flow & $6(37.4)$ & $15(60)$ & 0.09 \\
\hline 19 & Univentricular & $1(6.3)$ & $2(8)$ & 0.48 \\
\hline 20 & Redo surgery & $3(18.7)$ & $6(24)$ & 0.49 \\
\hline 21 & High-risk surgery & $5(31)$ & $18(72)$ & 0.02 \\
\hline 22 & $\begin{array}{l}\text { Cardiopulmonary bypass time in minutes } \\
\text { (mean } \pm \text { SD) }\end{array}$ & $153.71 \pm 101.88$ & $167.75 \pm 93.25$ & 0.51 \\
\hline 23 & Aorta cross clamp time in minutes (mean \pm SD) & $103.38 \pm 65.95$ & $105.82 \pm 66.46$ & 0.97 \\
\hline
\end{tabular}

Abbreviations: Pre-op, preoperative; SD, standard deviation.

such as sepsis, MV, previous surgery, chronic lung disease, neuromuscular disease, etc., were assessed in this study population. Sixteen (39\%) patients underwent ET and 25 (61\%) LT. Ten $(24.3 \%)$ children with transposition of great arteries (TGA) underwent arterial switch operation (ASO) and they constituted majority of the tracheostomized patients.

Postoperative complications, such as bleeding, reexploration, extracorporeal membrane oxygenation (ECMO) requirement, pressure sores, unanticipated cardiac arrest, accidental tracheostomy tube dislodgement, CNS injury, AKI, etc., are compared in - Table 3. Among the various postoperative complications, delayed sternal closure was the major morbidity (63\%), while ECMO requirement (4.8\%) was the least common complication.

About $31.7 \%$ of the children developed sepsis. VAP formed the major subset of HAI while CAUTI was the least in this cohort. Variables pertaining to HAI are compared in - Table 4. We evaluated the nutritional variables, such as days of nil per oral (NPO), days on total parenteral nutrition (TPN), days of enteral feed and feed interruption (FI), which are compared in - Table 5 .
Postoperative outcome variables are delineated in - Table 6. Various other following outcomes were compared between the two groups: day of tracheostomy initiation, days of tracheostomy MV, total days of MV, number of extubation trials, and tracheal complications following tracheostomy. In our cohort, the survival was $71 \%$, the major indication for tracheostomy was prolonged MV due to cardiac causes such as low cardiac output (LCO). The other indications were chylothorax, CNS events, diaphragmatic palsy, etc. Peritracheostomy clinical variables including hemodynamic, arterial blood gas (ABG), and laboratory parameters, positive end expiratory pressure (PEEP) requirement, and bleeding from the tracheostomy site are compared in - Table 7.

The current study identified many risk associations with the timing of tracheostomy in pediatric cardiac surgical patients. ET compared with LT had less risk associations with the following variables: incidences of preoperative hospital stay $(p=0.0016)$, preoperative sepsis $(p=0.03)$, high-risk surgery $(p=0.04)$, postoperative sepsis $(p=0.001)$, serum C-reactive protein (CRP; $p=0.04)$, total VAP ( $p=0.006)$, VAP before tracheostomy $(p=0.01)$, antibiotic escalation 
118 Tracheostomy in Postoperative Pediatric Cardiac Surgical Patients Bipin et al.

Table 3 Postcardiac surgical complications- comparison between early tracheostomy and late tracheostomy

\begin{tabular}{|c|c|c|c|c|}
\hline Sl. no. & $\begin{array}{l}\text { Clinical variables } \\
n(\%)\end{array}$ & $\begin{array}{l}\text { Early tracheostomy } \\
(n=16)\end{array}$ & $\begin{array}{l}\text { Late tracheostomy } \\
(n=25)\end{array}$ & $p$-Value \\
\hline 1 & Major bleeding needing intervention & $5(31)$ & $7(28)$ & 0.54 \\
\hline 2 & Re exploration & $4(25)$ & $13(52)$ & 0.08 \\
\hline \multirow[t]{3}{*}{3} & Cause of re exploration & & & \multirow[t]{3}{*}{0.27} \\
\hline & Bleeding & $4(25)$ & $7(28)$ & \\
\hline & Residual cardiac defect & $0(0)$ & $6(24)$ & \\
\hline 4 & DSC & $4(25)$ & $11(44)$ & 0.18 \\
\hline 5 & ECMO required & $0(0)$ & $2(8)$ & 0.36 \\
\hline 6 & CNS event & $5(31)$ & $7(28)$ & 0.54 \\
\hline \multirow[t]{3}{*}{7} & CNS event & & & \multirow[t]{3}{*}{0.68} \\
\hline & Seizure & $4(25)$ & $6(24)$ & \\
\hline & Low GCS & $1(6)$ & $1(4)$ & \\
\hline 8 & AKI & $5(31)$ & $11(44)$ & 0.31 \\
\hline 9 & Unanticipated arrest & $4(25)$ & $11(44)$ & 0.18 \\
\hline 10 & Accidental tube dislodgement & $1(6)$ & $5(20)$ & 0.22 \\
\hline 11 & Pressure sore & $1(6)$ & $11(44)$ & 0.01 \\
\hline
\end{tabular}

Abbreviations: AKI, acute kidney injury; CNS, central nervous system; DSC, delayed sternal closure; ECMO, extra corporeal membrane oxygenation; GCS, Glasgow coma scale.

Table 4 Comparison of postoperative clinical variables pertaining to infection between early tracheostomy and late tracheostomy

\begin{tabular}{|c|c|c|c|c|}
\hline Sl. no. & $\begin{array}{l}\text { Clinical variables } \\
n(\%)\end{array}$ & $\begin{array}{l}\text { Early tracheostomy } \\
(n=16)\end{array}$ & $\begin{array}{l}\text { Late tracheostomy } \\
(n=25)\end{array}$ & $p$-Value \\
\hline 1 & Sepsis & $1(6)$ & $12(48)$ & 0.006 \\
\hline \multirow[t]{11}{*}{2} & Pathogens isolated & & & \multirow[t]{11}{*}{0.71} \\
\hline & Pseudomonas & $1(6)$ & $1(4)$ & \\
\hline & Pseudomonas + Acinetobacter & $0(0)$ & $1(4)$ & \\
\hline & Klebsiella + Stenotrophomonas maltophilia & $0(0)$ & $1(4)$ & \\
\hline & Klebsiella & $0(0)$ & $3(12)$ & \\
\hline & Escherichia coli + Acinetobacter & $0(0)$ & $1(4)$ & \\
\hline & Proteus & $0(0)$ & $1(4)$ & \\
\hline & S. maltophila & $0(0)$ & $1(4)$ & \\
\hline & Acinetobacter & $0(0)$ & $1(4)$ & \\
\hline & Staphylococcus haemolyticus + Candida & $0(0)$ & $1(4)$ & \\
\hline & Klebsiella + Pseudomonas & $0(0)$ & $1(4)$ & \\
\hline 4 & CRP in $\mathrm{mg} / \mathrm{L}$ (mean $\pm \mathrm{SD}$ ) & $54.37 \pm 62.68$ & $84.33 \pm 50.36$ & 0.047 \\
\hline 5 & $\mathrm{PCT}$ in $\mathrm{ng} / \mathrm{mL}($ mean $\pm \mathrm{SD})$ & $44.34 \pm 63.96$ & $24.01 \pm 28.54$ & 0.48 \\
\hline 6 & Galactomannan in ODI (mean \pm SD) & $1.2 \pm 0.2$ & $0.98 \pm 0.60$ & 0.49 \\
\hline 7 & VAP total & $1(6)$ & $12(48)$ & 0.006 \\
\hline 8 & Pretracheostomy VAP & $0(0)$ & $8(32)$ & 0.01 \\
\hline 9 & Posttracheostomy VAP & $1(6)$ & $4(16)$ & 0.63 \\
\hline 10 & CRBSI & $0(0)$ & $3(12)$ & 0.21 \\
\hline 11 & CAUTI & $0(0)$ & $1(4)$ & 0.63 \\
\hline 12 & Superficial tracheal site infection & $2(12)$ & $6(24)$ & 0.31 \\
\hline 13 & SSI & $1(6)$ & $3(12)$ & 0.48 \\
\hline 14 & Antibiotic escalation & $5(30)$ & $19(76)$ & 0.006 \\
\hline 15 & Antifungal therapy & $2(12)$ & $13(52)$ & 0.018 \\
\hline
\end{tabular}

Abbreviations: CAUTI, catheter-associated Urinary tract infection; CRBSI, catheter-related blood stream infection; CRP, C-reactive protein; ODI, optical density index; PCT, procalcitonin; SD, standard deviation; SSI, surgical site infection; VAP, ventilator-associated pneumonia. 
Table 5 Clinical variables pertaining to postoperative nutrition compared between early tracheostomy and late tracheostomy

\begin{tabular}{|c|c|c|c|c|}
\hline SI. no. & $\begin{array}{l}\text { Clinical variables } \\
n(\%)\end{array}$ & $\begin{array}{l}\text { Early tracheostomy } \\
(n=16)\end{array}$ & $\begin{array}{l}\text { Late tracheostomy } \\
(n=25)\end{array}$ & p-Value \\
\hline 1 & Days on NPO (mean \pm SD) & $2.06 \pm 1.76$ & $3.32 \pm 3.61$ & 0.30 \\
\hline 2 & Days on TPN (mean \pm SD) & $3.16 \pm 6.01$ & $6.62 \pm 8.18$ & 0.27 \\
\hline 3 & Days of FI (mean \pm SD) & $2.69 \pm 4.17$ & $8.45 \pm 7.21$ & 0.0017 \\
\hline \multirow[t]{6}{*}{4} & Causes of $\mathrm{Fl}$ & & & \multirow[t]{6}{*}{0.30} \\
\hline & Seizure & $1(6)$ & $0(0)$ & \\
\hline & Surgical procedure & $1(6)$ & $5(20)$ & \\
\hline & Feed intolerance & $5(30)$ & $5(20)$ & \\
\hline & Surgical procedure + feed intolerance & $3(18)$ & $11(44)$ & \\
\hline & Seizure + feed intolerance & $1(6)$ & $3(12)$ & \\
\hline
\end{tabular}

Abbreviations: Fl, feed interruption; n, number of; NPO, nil per oral; SD, standard deviation; TPN, total parenteral nutrition.

Table 6 Comparison of postoperative outcome clinical variables between early and late tracheostomy

\begin{tabular}{|c|c|c|c|c|}
\hline SI. no. & Clinical variable & $\begin{array}{l}\text { Early tracheostomy } \\
(n=16)\end{array}$ & $\begin{array}{l}\text { Late tracheostomy } \\
(n=25)\end{array}$ & $p$-Value \\
\hline 1 & $\begin{array}{l}\text { Day of tracheostomy from MV initiation } \\
\text { (mean } \pm \text { SD) }\end{array}$ & $5.93 \pm 1.65$ & $17.48 \pm 11.66$ & 0.001 \\
\hline 2 & Days of endotracheal tube ventilation (mean \pm SD) & $6.26 \pm 1.03$ & $17.16 \pm 11.66$ & 0.001 \\
\hline 3 & $\begin{array}{l}\text { Days of tracheostomy tube ventilation } \\
\text { (mean } \pm \text { SD) }\end{array}$ & $16.18 \pm 15.94$ & $24.72 \pm 20.68$ & 0.36 \\
\hline 4 & Day of total MV (mean \pm SD) & $22.12 \pm 15.74$ & $41.4 \pm 24.78$ & 0.0027 \\
\hline 5 & No of trials of extubation (mean \pm SD) & $1 \pm 0.89$ & $1.16 \pm 0.98$ & 0.60 \\
\hline 6 & $\begin{array}{l}\text { No of times tracheostomy tube changed } \\
\text { (mean } \pm \text { SD) }\end{array}$ & $1.06 \pm 2.14$ & $2.24 \pm 2.61$ & 0.0210 \\
\hline \multirow[t]{5}{*}{7} & Causes for tracheostomy tube change & & & \multirow[t]{5}{*}{0.91} \\
\hline & Block & $1(6)$ & $3(12)$ & \\
\hline & Routine downsizing & $1(6)$ & $4(16)$ & \\
\hline & Accidental dislodgement & $1(6)$ & $1(4)$ & \\
\hline & Multiple & $3(18)$ & $11(44)$ & \\
\hline 8 & LOICUS (mean \pm SD) in days & $28.68 \pm 21.68$ & $46.76 \pm 25.74$ & 0.0143 \\
\hline 9 & LOHS (mean \pm SD) in days & $37.25 \pm 26.43$ & $66.28 \pm 38.02$ & 0.0014 \\
\hline 10 & Total ventilation-free days (mean \pm SD) & $16.31 \pm 12.97$ & $32.76 \pm 29.83$ & 0.0272 \\
\hline 11 & Decannulated in ICU & $14(88)$ & $19(76)$ & 0.20 \\
\hline 12 & $\begin{array}{l}\text { Duration of tracheal cannulation in days (mean } \pm \\
\text { SD) }\end{array}$ & $19.45 \pm 6.91$ & $31.46 \pm 20.92$ & 0.0354 \\
\hline \multirow[t]{5}{*}{13} & Tracheostomy complication & $3(18)$ & $10(40)$ & \multirow[t]{5}{*}{0.13} \\
\hline & Tracheal stenosis & $1(6)$ & $5(20)$ & \\
\hline & TBM & $1(6)$ & $0(0)$ & \\
\hline & Granulation & $1(6)$ & $4(16)$ & \\
\hline & Tracheal rent & $0(0)$ & $1(4)$ & \\
\hline 14 & Discharged & $13(81)$ & $16(64)$ & 0.20 \\
\hline 15 & Day of death (mean \pm SD) & $21 \pm 8.54$ & $60.77 \pm 35.27$ & 0.052 \\
\hline 16 & Discharged with tracheostomy in situ & $2(12)$ & $5(20)$ & 0.29 \\
\hline 17 & Day of discharge (mean \pm SD) & $39.69 \pm 28.37$ & $66.37 \pm 41.02$ & 0.0089 \\
\hline \multirow[t]{3}{*}{18} & Indication for tracheostomy & & & \multirow[t]{3}{*}{$<0.001$} \\
\hline & Prolonged ventilation & $7(42)$ & $25(100)$ & \\
\hline & Easy wean from ventilator & $9(54)$ & $0(0)$ & \\
\hline
\end{tabular}


Table 6 (Continued)

\begin{tabular}{|c|c|c|c|c|}
\hline SI. no. & Clinical variable & $\begin{array}{l}\text { Early tracheostomy } \\
(n=16)\end{array}$ & $\begin{array}{l}\text { Late tracheostomy } \\
(n=25)\end{array}$ & $p$-Value \\
\hline \multirow[t]{15}{*}{19} & Reason for prolonged ventilation & & & \multirow[t]{15}{*}{0.54} \\
\hline & Cardiac & $1(6)$ & $8(32)$ & \\
\hline & TBM & $0(0)$ & $0(0)$ & \\
\hline & Diaphragmatic palsy & $0(0)$ & $0(0)$ & \\
\hline & CNS event & $0(0)$ & $0(0)$ & \\
\hline & Chylothorax & $0(0)$ & $0(0)$ & \\
\hline & SGS/granulation & $0(0)$ & $0(0)$ & \\
\hline & Cardiac + TBM & $0(0)$ & $1(4)$ & \\
\hline & Cardiac + diaphragmatic palsy & $0(0)$ & $1(4)$ & \\
\hline & Cardiac + CNS event + chylothorax & $4(24)$ & $3(12)$ & \\
\hline & Cardiac + CNS event & $1(6)$ & $2(8)$ & \\
\hline & Cardiac + chylothorax & $1(6)$ & $9(36)$ & \\
\hline & Cardiac + SCS & $0(0)$ & $1(4)$ & \\
\hline & Cardiac + SCS + CNS event & $0(0)$ & $0(0)$ & \\
\hline & Multiple & $0(0)$ & $0(0)$ & \\
\hline
\end{tabular}

Abbreviations: CNS, central nervous system; ICU, intensive care unit; LOHS, length of hospital stay; LOICUS, length of ICU stay; SD, standard deviation; SGS, subglottic stenosis; TBM, tracheobronchomalacia.

Table 7 Comparison of peritracheostomy clinical variables between Early and Late Tracheostomy

\begin{tabular}{|c|c|c|c|c|c|c|c|c|c|c|c|c|c|}
\hline \multirow[t]{2}{*}{ SI. no. } & \multirow{2}{*}{$\begin{array}{l}\text { Variable } \\
\text { (mean } \pm \\
\text { SD) }\end{array}$} & \multicolumn{3}{|c|}{ Before TT } & \multicolumn{3}{|c|}{ Tracheostomy day 1} & \multicolumn{3}{|c|}{ Tracheostomy day 2} & \multicolumn{3}{|c|}{ Tracheostomy day 3} \\
\hline & & ET & LT & $p$ & ET & LT & $p$ & ET & L T & $p$ & ET & LT & $p$ \\
\hline 1 & $\mathrm{PH}$ & $\begin{array}{l}7.30 \pm \\
0.16\end{array}$ & $\begin{array}{l}7.39 \pm \\
0.08\end{array}$ & 0.03 & $\begin{array}{l}7.40 \pm \\
0.08\end{array}$ & $\begin{array}{l}7.41 \pm \\
0.06\end{array}$ & 0.83 & $\begin{array}{l}7.40 \pm \\
0.066\end{array}$ & $\begin{array}{l}7.40 \pm \\
0.060\end{array}$ & 0.78 & $\begin{array}{l}7.41 \pm \\
0.02\end{array}$ & $\begin{array}{l}7.38 \pm \\
0.05\end{array}$ & 0.15 \\
\hline 2 & $\mathrm{PCO}_{2}$ & $\begin{array}{l}57.1 \pm \\
38.8\end{array}$ & $\begin{array}{l}42.5 \pm \\
14.2\end{array}$ & 0.68 & $\begin{array}{l}38.5 \pm \\
14.5\end{array}$ & $\begin{array}{l}39.2 \pm \\
9.3\end{array}$ & 0.27 & $\begin{array}{l}35.2 \pm \\
6.8\end{array}$ & $\begin{array}{l}39.3 \pm \\
9.7\end{array}$ & 0.23 & $\begin{array}{l}36.7 \pm \\
6.1\end{array}$ & $\begin{array}{l}41.1 \pm \\
11.1\end{array}$ & 0.33 \\
\hline 3 & $\mathrm{PO}_{2}$ & $\begin{array}{l}90.6 \pm \\
62.4\end{array}$ & $\begin{array}{l}119.3 \pm \\
91.1\end{array}$ & 0.23 & $\begin{array}{l}115.5 \pm \\
52.6\end{array}$ & $\begin{array}{l}111.4 \pm \\
59.8\end{array}$ & 0.7 & $\begin{array}{l}127.6 \pm \\
61.6\end{array}$ & $\begin{array}{l}104 \pm \\
59.5\end{array}$ & 0.23 & $\begin{array}{l}135 \pm \\
75.2\end{array}$ & $\begin{array}{l}103 \pm \\
56.7\end{array}$ & 0.19 \\
\hline 4 & BE & $\begin{array}{l}0.51 \pm \\
5.051\end{array}$ & $\begin{array}{l}0.03 \pm \\
3.63\end{array}$ & 0.60 & $\begin{array}{l}0.16 \pm \\
3.5\end{array}$ & $\begin{array}{l}0.22 \pm \\
3.75\end{array}$ & 0.9 & $\begin{array}{l}0.69 \pm \\
4.4\end{array}$ & $\begin{array}{l}0.004 \pm \\
4.3\end{array}$ & 0.57 & $\begin{array}{l}0.14 \pm \\
4.04\end{array}$ & $\begin{array}{l}0.14 \pm \\
3.9\end{array}$ & 0.95 \\
\hline 5 & $\mathrm{HCO}_{3}$ & $\begin{array}{l}24.8 \pm \\
6.4\end{array}$ & $\begin{array}{l}24.6 \pm \\
3.6\end{array}$ & 0.87 & $\begin{array}{l}24.3 \pm \\
4.3\end{array}$ & $\begin{array}{l}24.1 \pm \\
3.4\end{array}$ & 0.89 & $\begin{array}{l}23.1 \pm \\
4.2\end{array}$ & $\begin{array}{l}24.2 \pm \\
3.8\end{array}$ & 0.39 & $\begin{array}{l}24.0 \pm \\
4.4\end{array}$ & $\begin{array}{l}23.9 \pm \\
3.4\end{array}$ & 0.96 \\
\hline 6 & $H B$ & $\begin{array}{l}12.4 \pm \\
1.6\end{array}$ & $\begin{array}{l}11.5 \pm \\
1.2\end{array}$ & 0.04 & $\begin{array}{l}12.2 \pm \\
1.5\end{array}$ & $\begin{array}{l}11.6 \pm \\
1.6\end{array}$ & 0.26 & $\begin{array}{l}12.5 \pm \\
1.6\end{array}$ & $\begin{array}{l}11.6 \pm \\
1.3\end{array}$ & 0.04 & $\begin{array}{l}12.2 \pm \\
1.2\end{array}$ & $\begin{array}{l}11.1 \pm \\
0.8\end{array}$ & 0.001 \\
\hline 7 & PCV & $\begin{array}{l}38.1 \pm \\
4.4\end{array}$ & $\begin{array}{l}34.8 \pm \\
3.4\end{array}$ & 0.01 & $\begin{array}{l}36.8 \pm \\
4.1\end{array}$ & $\begin{array}{l}33.2 \pm \\
8.0\end{array}$ & 0.03 & $\begin{array}{l}39.2 \pm \\
3.5\end{array}$ & $\begin{array}{l}34.0 \pm \\
5.8\end{array}$ & 0.001 & $\begin{array}{l}38.7 \pm \\
2.6\end{array}$ & $\begin{array}{l}33.4 \pm \\
5.6\end{array}$ & 0.001 \\
\hline 8 & $\mathrm{SAO}_{2}$ & $\begin{array}{l}83.1 \pm \\
23.1\end{array}$ & $\begin{array}{l}93.3 \pm \\
9.7\end{array}$ & 0.06 & $\begin{array}{l}93.4 \pm \\
10.8\end{array}$ & $\begin{array}{l}94.2 \pm \\
7.5\end{array}$ & 0.72 & $\begin{array}{l}94.5 \pm \\
10.5\end{array}$ & $\begin{array}{l}92.6 \pm \\
9.2\end{array}$ & 0.55 & $\begin{array}{l}97.2 \pm \\
3.6\end{array}$ & $\begin{array}{l}93.8 \pm \\
7.1\end{array}$ & 0.09 \\
\hline 9 & $H R$ & $\begin{array}{l}124.4 \pm \\
17.2\end{array}$ & $\begin{array}{l}127.8 \pm \\
20.0\end{array}$ & 0.58 & $\begin{array}{l}128.2 \pm \\
18.3\end{array}$ & $\begin{array}{l}128.7 \pm \\
15.9\end{array}$ & 0.9 & $\begin{array}{l}128.7 \pm \\
13.9\end{array}$ & $\begin{array}{l}125 \pm \\
13.7\end{array}$ & 0.48 & $\begin{array}{l}127.0 \pm \\
13\end{array}$ & $\begin{array}{l}120 \pm \\
17.9\end{array}$ & 0.18 \\
\hline 10 & SBP & $\begin{array}{l}93.3 \pm \\
13.0\end{array}$ & $\begin{array}{l}81.3 \pm \\
16.2\end{array}$ & 0.01 & $\begin{array}{l}87.6 \pm \\
15.2\end{array}$ & $\begin{array}{l}88.3 \pm \\
18.2\end{array}$ & 0.9 & $\begin{array}{l}90.3 \pm \\
16.9\end{array}$ & $\begin{array}{l}87.6 \pm \\
16.5\end{array}$ & 0.62 & $\begin{array}{l}89.1 \pm \\
18.0\end{array}$ & $\begin{array}{l}87.1 \pm \\
18.0\end{array}$ & 0.72 \\
\hline 11 & DBP & $\begin{array}{l}57.9 \pm \\
6.6\end{array}$ & $\begin{array}{l}49.1 \pm \\
12.1\end{array}$ & 0.01 & $\begin{array}{l}56.6 \pm \\
11.1\end{array}$ & $\begin{array}{l}55.8 \pm \\
15.9\end{array}$ & 0.72 & $\begin{array}{l}60.6 \pm \\
12.5\end{array}$ & $\begin{array}{l}54.4 \pm \\
13.6\end{array}$ & 0.85 & $\begin{array}{l}57.8 \pm \\
9.6\end{array}$ & $\begin{array}{l}53 \pm \\
10.3\end{array}$ & 0.14 \\
\hline 12 & RA & $\begin{array}{l}13.0 \pm \\
4.9\end{array}$ & $\begin{array}{l}10.5 \pm \\
5.2\end{array}$ & 0.13 & $\begin{array}{l}12.5 \pm \\
5.5\end{array}$ & $\begin{array}{l}12.2 \pm \\
5.9\end{array}$ & 0.8 & $\begin{array}{l}12.8 \pm \\
5.8\end{array}$ & $\begin{array}{l}11.3 \pm \\
6.2\end{array}$ & 0.51 & $\begin{array}{l}11.3 \pm \\
6.2\end{array}$ & $\begin{array}{l}11.3 \pm \\
5.5\end{array}$ & 0.9 \\
\hline 13 & LAC & $1.9 \pm 2.6$ & $1.4 \pm 1.2$ & 0.54 & $1.5 \pm 3.2$ & $1.3 \pm 0.7$ & 0.32 & $2.1 \pm 3.4$ & $1.3 \pm 1.1$ & 0.64 & $1.6 \pm 1.8$ & $1.3 \pm 1.1$ & 0.8 \\
\hline
\end{tabular}


Table 7 (Continued)

\begin{tabular}{|c|c|c|c|c|c|c|c|c|c|c|c|c|c|}
\hline \multirow[t]{2}{*}{ SI. no. } & \multirow{2}{*}{$\begin{array}{l}\text { Variable } \\
\text { (mean } \pm \\
\text { SD) }\end{array}$} & \multicolumn{3}{|c|}{ Before TT } & \multicolumn{3}{|c|}{ Tracheostomy day 1} & \multicolumn{3}{|c|}{ Tracheostomy day 2} & \multicolumn{3}{|c|}{ Tracheostomy day 3} \\
\hline & & ET & LT & $p$ & ET & LT & $p$ & ET & L T & $p$ & ET & LT & $p$ \\
\hline 14 & RBS & $\begin{array}{l}110.0 \pm \\
27.1\end{array}$ & $\begin{array}{l}112.6 \pm \\
38.2\end{array}$ & 0.86 & $\begin{array}{l}150.1 \pm \\
120.2\end{array}$ & $\begin{array}{l}113.3 \pm \\
27.2\end{array}$ & 0.14 & $\begin{array}{l}106.8 \pm \\
19.6\end{array}$ & $\begin{array}{l}112 \pm \\
31.2\end{array}$ & 0.57 & $\begin{array}{l}112 \pm \\
53.4\end{array}$ & $\begin{array}{l}115 \pm \\
30.4\end{array}$ & 0.54 \\
\hline 15 & VIS & $\begin{array}{l}57.8 \pm \\
170.8\end{array}$ & $\begin{array}{l}20.5 \pm \\
16.1\end{array}$ & 0.66 & $\begin{array}{l}141.5 \pm \\
504.5\end{array}$ & $\begin{array}{l}18.5 \pm \\
14.2\end{array}$ & 0.63 & $\begin{array}{l}10.8 \pm \\
9.3\end{array}$ & $\begin{array}{l}14.6 \pm \\
12.9\end{array}$ & 0.51 & $\begin{array}{l}10.8 \pm \\
9.3\end{array}$ & $\begin{array}{l}14.6 \pm \\
12.9\end{array}$ & 0.51 \\
\hline 16 & CFB & $\begin{array}{l}22.4 \pm \\
122.7\end{array}$ & $\begin{array}{l}154.5 \pm \\
280.5\end{array}$ & 0.18 & $\begin{array}{l}44.2 \pm \\
129.9\end{array}$ & $\begin{array}{l}191 \pm \\
296.2\end{array}$ & 0.06 & $\begin{array}{l}76.8 \pm \\
133.2\end{array}$ & $\begin{array}{l}213 \pm \\
330\end{array}$ & 0.53 & $\begin{array}{l}95.7 \pm \\
150\end{array}$ & $\begin{array}{l}239 \pm \\
354\end{array}$ & 0.94 \\
\hline 17 & PT & $\begin{array}{l}15.25 \pm \\
1.48\end{array}$ & $\begin{array}{l}16.17 \pm \\
1.01\end{array}$ & 0.09 & & & & & & & & & \\
\hline 18 & APTT & $\begin{array}{l}33.64 \pm \\
3.05\end{array}$ & $\begin{array}{l}40.15 \pm \\
10.30\end{array}$ & 0.71 & & & & & & & & & \\
\hline 19 & INR & $\begin{array}{l}1.29 \pm \\
0.14\end{array}$ & $\begin{array}{l}1.80 \pm \\
1.17\end{array}$ & 0.04 & & & & & & & & & \\
\hline 20 & PLT & $\begin{array}{l}111.45 \pm \\
109.52\end{array}$ & $\begin{array}{l}100.89 \pm \\
80.20\end{array}$ & 0.52 & & & & & & & & & \\
\hline 21 & PEEP & $\begin{array}{l}4.5 \pm \\
0.51\end{array}$ & $4.96 \pm$ & 0.03 & & & & & & & & & \\
\hline 22 & $\begin{array}{l}\text { BLD } \\
n \%\end{array}$ & $0(0)$ & $3(12)$ & 0.21 & & & & & & & & & \\
\hline
\end{tabular}

Abbreviations: APTT, activated partial thromboplastin time in seconds; BE, base excess in meq/L; BLD, bleeding from tracheostomy site: no bleeding; CFB, cumulative fluid balance in $\mathrm{mL} / \mathrm{kg}$;; $\mathrm{ET}$, early tracheostomy; $\mathrm{HB}$, hemoglobin in $\mathrm{g} / \mathrm{dL} ; \mathrm{HCO}_{3}$, bicarbonate in meq/L; $\mathrm{Hg} \mathrm{DBP}$, diastolic blood pressure in $\mathrm{mm} \mathrm{Hg}$; HR, heart rate in per minute; INR, international normalized ratio; LAC, lactate in mmol/L; LT, Late tracheostomy; $\mathrm{PCO}_{2}$, partial pressure of $\mathrm{CO}_{2}$ in $\mathrm{mm} \mathrm{Hg}$; $\mathrm{PCV}$, hematocrit in \%; $\mathrm{PLT}$, platelet count in $\times 1,000 / \mathrm{mL}$; $\mathrm{PO}_{2}$, partial pressure of $\mathrm{O}_{2}$ in mm $\mathrm{Hg}$; PT, prothrombin time in seconds; $\mathrm{RA}$, right atrial pressure in $\mathrm{mm} \mathrm{Hg} ; \mathrm{RBS}$, random blood sugar mg/dl; $\mathrm{SAO}_{2}$, Saturation of $\mathrm{O}_{2}$ in \%; $\mathrm{SBP}$, systolic blood pressure in $\mathrm{mm}$; SD, standard deviation; VIS, vasotrope inotropic score.

$(p=0.006)$ and antifungal therapy $(p=0.01)$ requirement, pressure sores $(p=0.01)$, and international normalized ratio (INR) before tracheostomy ( $p=0.04$ ).

Furthermore, ET was associated with lesser days of FI $(p=0.0017), \operatorname{MV}(p=0.0027)$, LOICUS $(p=0.01)$, and LOHS $(p=0.001)$, lesser PEEP requirement before tracheostomy $(p=0.03)$, less tracheostomy tube change $(p=0.02)$. These groups of children were decannulated faster $(p=$ $0.03)$ and discharged earlier $(p=0.0089)$.

\section{Discussion}

This prospective study on the timing of tracheostomy described the differences in terms of benefits and drawbacks between ET and LT. However, we didn't come across any such prospective study on this subject in children undergoing congenital cardiac repair.

\section{Mortality}

We, in our study, did not find statistically significant difference in mortality between ET and LT groups. Similar observations were made in their studies by Griffiths et al, ${ }^{14}$ Huang et al (a meta-analysis involving nine randomized controlled trials [RCTs] which included 2,072 patients) $)^{15}$ and Young et al (TracMan study). ${ }^{16}$ Trouillet and colleague in their RCT involving CSICU patients didn't find significant difference in mortality between ET and LT. ${ }^{17}$ On the contrary, Rumbak et al showed a significant reduction in mortality (31.7 vs. $61.7 \%$ ) in their RCT of ET (48 hours) versus LT (14-16 days) in medical ICU patients. ${ }^{18}$ The authors in a large cohort of 11,000 patients requiring tracheostomy over a period of 12 years found that each additional day of delaying tracheostomy was associated with increased long-term mortality. ${ }^{19}$ Shaw and Santry in a retrospective cohort of 49,191 patients, compared ET ( $<7$ days) versus LT ( $>10$ days) and mortality was lower in ET group (14\% ET vs. $21 \% \mathrm{LT} ; p<0.0001) .{ }^{20}$ Survival advantage with ET was demonstrated by other authors like Yavas et al (in 205 CSICU patients) $)^{21}$ and Devarajan et al (who demonstrated lower mortality [21.1\% ET vs. $40.4 \%$ LT] and cardiac morbidity [14\% ET vs. 33\% LT] in ET group). ${ }^{22}$ Hosseinian et al, in their study in adult patients with respiratory failure, found that ET reduces mortality in comparison to $\mathrm{LT}^{23}$

\section{Pre and Intraoperative Variables}

ET group was associated with shorter prehospital stay $(p=0.0016)$ and lesser preoperative sepsis $(p=0.03)$. In our center, we treat those on sepsis before surgery except few with some emergencies like infective endocarditis and intracardiac shunt with heart failure. The indication for tracheostomy in our cohort was mostly predicted prolong MV.

LT group was having statistically significant risk association of high-risk surgical procedures $(p=0.042)$. We feel that the complex cardiac pathologies requiring a high-risk procedures were having significant comorbidities in the 
preoperative period, such as sepsis and hospitalization, MV, and this may be the reason why they had to undergo tracheostomy. Conforti et al in their study found that infants with congenital airway anomalies more frequently required ET $(12 / 52,23 \%$ vs. $0 / 124,0 \% ; p=0.0001) .{ }^{24}$ We did not make any such observation in our study cohort.

\section{Postoperative Complications}

In our study, ET was associated with statistically significant lower pressure sores in the postoperative period $(p=0.01)$. Supporting our finding, studies in traumatic brain injury patients have found that tracheostomy in proper timing reduced pressure ulcer incidence. . $5,26^{2}$

\section{Postoperative Infection}

In our study, we demonstrated a lesser association of postoperative sepsis $(p=0.001)$, lower CRP levels $(p=0.04)$, less antibiotic escalation $(p=0.006)$, and antifungal therapy $(p=0.01)$ requirement in the ET group. There was statistically significant association of lower total $\operatorname{VAP}(p=0.006)$ and pretracheostomy VAP $(p=0.01)$ rates in the ET group. Similar to our observation, Rumbak et al noticed lower incidence of pneumonia in their ET versus LT study (5 vs. $25 \%) .{ }^{18}$ Shaw and Santry also conformed a significant difference in VAP (12\% ET vs. $15 \%$ LT; $p$ $<0.0001){ }^{20}$ However, Huang et al did not find significant difference of VAP in ET patients. ${ }^{15}$ According to Trouillet et al, VAP, as well as incidence of other infections were similar between the ET and LT groups. ${ }^{17}$ Yavas et al quoted that ET was associated with lower incidence of infections. ${ }^{21}$ Ben Avi et al reported lower incidence of SSI with ET (1.11\% ET vs. 8.26\% LT). ${ }^{27}$

\section{Postoperative Nutrition}

Yavas et al opined that ET was associated with earlier enteral feeding. ${ }^{21}$ Our study was in line with their finding and we found statistically significant less FI with ET ( $p=0.001)$.

\section{Postoperative Outcomes}

Our study concluded that there was significant association of lesser LOICUS $(p=0.01)$ and LOHS $(p=0.001)$, earlier discharge ( $p=0.008)$ with ET. Our findings corroborated with other studies by Griffiths et al, ${ }^{14}$ and Yavas et al, ${ }^{21}$ and Shaw and Santry (LOICUS: 16 days ET vs. 27 days LT, $p<0.0001$; and LOHS: 25 days ET vs. 38 days LT, $p<0.0001){ }^{20}$

In our study, we noticed a statistically significant difference between the two groups with respect to the day of tracheostomy initiation $(p=0.001)$, days of endotracheal tube ventilation $(p=0.001)$, total days of MV $(p=0.002)$, number of times tracheostomy tube needed a change $(p=0.02)$, day of decannulation $(p=0.03$ ), and total ventilation-free days $(p=0.02)$. However, the other authors like Huang et $\mathrm{al},{ }^{15}$ Ben Avi et al, ${ }^{27}$ and Trouillet et al ${ }^{17}$ did not find significantly difference between ET and LT in terms of duration of MV and other ventilation parameters as opposed to our finding. But according to Trouillet et al, patients within the ET group required less sedation and experienced greater comfort of ICU stay and earlier resumption of patient autonomy. ${ }^{17}$

\section{Peritracheostomy Variables}

The coagulation parameters, like activated partial thromboplastin time (aPTT) and platelet count, were appropriately maintained during the peritracheostomy period in both the groups but INR was significantly lower for ET group $(p=0.04)$. This is because the earlier the procedure done, the lesser were the inflammation-, infection-, and transfusion-related coagulation derangement in comparison with LT, giving a safer edge for conducting the procedure without complications. ET was associated with a significantly higher hematocrits at various time points. This may be explained by the ongoing blood losses during the postoperative period, iatrogenic repeated blood sampling, and perioperative anemia in LT group. Similarly systolic and diastolic blood pressures were favorably maintained in ET compared with LT group. ET was also found to be associated with a lower PEEP requirement $(p=0.03)$.

\section{Study Limitations}

Our study has several limitations. Its observational nature precludes us from drawing any causality between the survival and perioperative risk factors and other morbidities. Though we did not perform a formal cost analysis but reductions in ICU and hospital stay is always associated with considerable cost savings and resource optimization. The number of patients who underwent tracheostomy is quite low when compared with other studies. Another limitation is the inability to assess intermediate and long-term outcomes.

\section{Conclusion}

Our study on postoperative pediatric cardiac patients demonstrated that ET can be safely performed with no increased risk of sternal wound infection or sepsis. There are significant advantages with ET in terms of reduction in postoperative morbidities with overall shorter duration of MV, ICU, and hospital stay which led to better enteral nutrition, faster convalescence, earlier tracheal decannulation, and patient discharge. These benefits ultimately promote faster patient rehabilitation with reduced healthcare costs.

\section{Funding \\ None.}

\section{Conflict of Interest}

None declared.

\section{References}

1 Mastropietro CW, Benneyworth BD, Turrentine M, et al. Tracheostomy after operations for congenital heart disease: an analysis of the society of thoracic surgeons congenital heart surgery database. Ann Thorac Surg 2016;101(6):2285-2292

2 Armstrong PA, McCarthy MC, Peoples JB. Reduced use of resources by early tracheostomy in ventilator-dependent patients with blunt trauma. Surgery 1998;124(4):763-766, discussion 766-767

3 Combes A, Luyt CE, Nieszkowska A, Trouillet JL, Gibert C, Chastre J. Is tracheostomy associated with better outcomes 
for patients requiring long-term mechanical ventilation? Crit Care Med 2007;35(3):802-807

4 Rodriguez JL, Steinberg SM, Luchetti FA, Gibbons KJ, Taheri PA, Flint LM. Early tracheostomy for primary airway management in the surgical critical care setting. Surgery 1990;108(4):655-659

5 MacIntyre NR, CookDJ, Ely EWJr, et al; American College of Chest PhysiciansAmerican Association for Respiratory CareAmerican College of Critical Care Medicine. Evidence-based guidelines for weaning and discontinuing ventilatory support: a collective task force facilitated by the American College of Chest Physicians; the American Association for Respiratory Care; and the American College of Critical Care Medicine. Chest 2001;120(6,suppl) :375S-395S

6 Gupta S, Dixit S, Choudhry D, et al. Tracheostomy in adult intensive care unit: an ISCCM expert panel practice recommendation. Indian J Crit Care Med 2020;24(suppl 1) :S31-S42

7 MacIntyre NR, Epstein SK, Carson S, Scheinhorn D, Christopher K, Muldoon S; National Association for Medical Direction of Respiratory Care. Management of patients requiring prolonged mechanical ventilation: report of a NAMDRC consensus conference. Chest 2005;128(6):3937-3954

8 Rahmanian PB, Adams DH, Castillo JG, Chikwe J, Filsoufi F. Tracheostomy is not a risk factor for deep sternal wound infection after cardiac surgery. Ann Thorac Surg 2007;84(6):1984-1991

9 Wouters R, Wellens F, Vanermen H. De Geest R, Degrieck I, De Meerleer F. Sternitis and mediastinitis after coronary artery bypass grafting. Analysis of risk factors. Tex Heart Inst J 1994;21(3):183-188

10 Peinado DMC, Bedrinana JIC. Comparison of NCHS-1977, CDC-2000 and WHO-2006 Nutritional Classification in 32 to 60 month-old Children in the Central Highlands of Peru (1992-2007). Univers J Public Health 2013;1(3):143-149

11 Kasiske B, Eckardt K. Kidney disease: improving global outcomes (KDIGO) acute kidney injury work group. KDIGO clinical practice guideline for acute kidney injury. Kidney inter. Suppl. 2012;2:1-138

12 Singer M, Deutschman CS, Seymour CW, et al. The third international consensus definitions for sepsis and septic shock (Sepsis-3) JAMA 2016;315(8):801-810

13 Centers for Disease Control and Prevention/National Healthcare Safety Network. CDC/NHSN surveillance definitions for specific types of infections. Available at: https://www.cdc.gov/ nhsn/pdfs/pscmanual/2020pscnosinfdef_current.pdf. Accessed January 15,2021

14 Griffiths J, Barber VS, Morgan L, Young JD. Systematic review and meta-analysis of studies of the timing of tracheostomy in adult patients undergoing artificial ventilation. BMJ 2005;330(7502):1243

15 Huang $\mathrm{H}$, Li Y, Ariani F, Chen X, Lin J. Timing of tracheostomy in critically ill patients: a meta-analysis. PLoS One 2014;9(3):e92981

16 Young D, Harrison DA, Cuthbertson BH, Rowan K; TracMan Collaborators. Effect of early vs late tracheostomy placement on survival in patients receiving mechanical ventilation: the TracMan randomized trial. JAMA 2013;309(20):2121-2129

17 Trouillet JL, Luyt CE, Guiguet M, et al. Early percutaneous tracheotomy versus prolonged intubation of mechanically ventilated patients after cardiac surgery: a randomized trial. Ann Intern Med 2011;154(6):373-383

18 Rumbak MJ, Newton M, Truncale T, Schwartz SW, Adams JW, Hazard PB. A prospective, randomized, study comparing early percutaneous dilational tracheotomy to prolonged translaryngeal intubation (delayed tracheotomy) in critically ill medical patients. Crit Care Med 2004;32(8):1689-1694

19 Scales DC, Thiruchelvam D, Kiss A, Redelmeier DA. The effect of tracheostomy timing during critical illness on long-term survival. Crit Care Med 2008;36(9):2547-2557

20 Shaw JJ, Santry HP. Who gets early tracheostomy?: evidence of unequal treatment at 185 academic medical centers. Chest 2015;148(5):1242-1250

21 Yavas S, Yagar S, Mavioglu L, et al. Tracheostomy: how and when should it be done in cardiovascular surgery ICU? J Card Surg 2009;24(1):11-18

22 Devarajan J, Vydyanathan A, Xu M, et al. Early tracheostomy is associated with improved outcomes in patients who require prolonged mechanical ventilation after cardiac surgery. J Am Coll Surg 2012;214(6):1008-16.e4

23 Hosseinian L, Chiang Y, Itagaki S, Polanco A, Rhee A, Chikwe J. Earlier versus later tracheostomy in patients with respiratory failure after cardiac surgery in the United States. J Cardiothorac Vasc Anesth 2014;28(3):488-492

24 Conforti A, Valfrè L, Scuglia M, et al. Laryngotracheal abnormalities in esophageal atresia patients: a hidden entity. Front Pediatr 2018;6:401

25 Alali AS, Scales DC, Fowler RA, et al. Tracheostomy timing in traumatic brain injury: a propensity-matched cohort study. J Trauma Acute Care Surg 2014;76(1):70-76, discussion 76-78

26 Babu A, Madhavan K, Singhal M, Sagar S, Ranjan P. Pressure ulcer surveillance in neurotrauma patients at a level one trauma centre in India. Oman Med J 2015;30(6):441-446

27 Ben-Avi R, Ben-Nun A, Levin S, et al. Tracheostomy after cardiac surgery: timing of tracheostomy as a risk factor for mortality. J Cardiothorac Vasc Anesth 2014;28(3):493-496 